\title{
DESIGN OF AN ELECTROSTATIC ENERGY SEPARATOR FOR THE ISIS RFQ TEST STAND
}

\author{
J. Duke, A. Letchford, RAL, Chilton, Didcot, UK
}

\begin{abstract}
It is planned to replace the $665 \mathrm{keV}$ Cockroft Walton set of the ISIS spallation neutron source in the UK with a four rod RFQ. Before this replacement is made, the performance of the RFQ will be extensively assessed on a test stand. One of the quantities we hope to measure is the proportion of accelerated ( trapped) beam at the exit of the RFQ for various $\mathrm{H}^{-}$ion source currents and rod voltages. In order to do this it is necessary to separate the untrapped beam which is largely at the ion source extraction energy of $35 \mathrm{keV}$ from the trapped beam which has an energy centred on $665 \mathrm{keV}$. This paper describes the design process for an instrument to perform this separation using parallel electrostatic plates to bend away the low energy beam while allowing all the higher energy beam to pass through, therefore measuring directly the fraction of trapped beam, and by implication the fraction of untrapped beam.
\end{abstract}

\section{THE DESIGN PROCESS}

The aim of the investigation was to find a method of removing the low energy component from the beam while retaining the high energy component for long enough that its current could be measured. Initially, extensive studies were performed to discover if it was possible to use the chromaticity of a series of quadrupoles to over focus the low energy beam and cause it to hit the beam pipe while retaining the high energy beam. ( For the purposes of this paper, 'high energy' is defined as $0.665 \pm 0.1 \mathrm{MeV}$ and low energy is anything less than this ). Solutions were found, but due to the highly divergent beam from the RFQ the beam size grows large very quickly, making it necessary to have very large bore quadrupoles in order not to lose any high energy beam. The size, weight and cost of these quadrupoles caused us to look for another way of separating the two energy groups. The fact that the untrapped beam is at such low energy and that there is a wide gap between the two energy groups caused us to think of using an electrostatic method of separating the groups.

Studies were performed to investigate the feasibility of using two parallel high voltage plates of opposite polarity to deflect the low energy beam more than the high energy beam. These were done by tracking a 'large' distribution of quasi-particles which had been generated by an RFQ simulation program written by A. Letchford. The final optimisation was performed using an initial distribution of 95351 quasi-particles, of which 89349 were 'high energy' and 6002 of lower energy, which corresponds to fractions of $93.7 \%$ and $6.3 \%$ respectively. Particle tracking was performed with Parmila [1] throughout, and various Fortran routines were written to perform tasks such as counting the proportions in the two energy groups at various stages of the device, calculating the trajectories of all the particles as they pass through the electric field and calculating the distribution of particles hits on the plate which the particles are bent towards.

The parallel plate device was to be situated in the second half of a diagnostics box of outside length 430 $\mathrm{mm}$, the first half of which will contain an emittance scanner. The entrance port of the box ends $163 \mathrm{~mm}$ after the end of the RFQ rods. At this point the low energy beam is already being lost on the $70 \mathrm{~mm}$ diameter beam pipe, and all components of the beam are diverging. At the entrance port to the box, the high energy component is quite round with a radius in both transverse directions of about $8 \mathrm{~mm}$. By the middle of the box, e.g. after $253 \mathrm{~mm}$ from the port, the beam looks transversely like Figure 1.

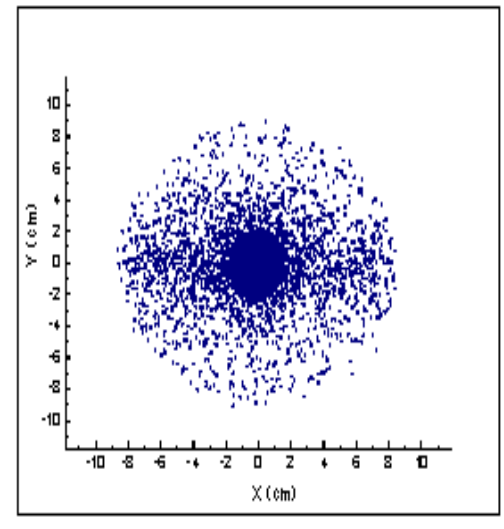

Fig. 1

Transverse distribution of all remaining particles at position of beginning of parallel plates, if there were no aperture plate

The radius in the $x$ direction of the high energy beam only at the same point in the box is about $1.5 \mathrm{~cm}$. This means that if nothing else were done the low energy beam would have to be bent $\sim(1.5+8.0) \mathrm{cm}$ more than the high energy beam to be spatially separated.

For this reason an aperture plate was added to the middle of the box, $217 \mathrm{~mm}$ after the entrance port, to crop the low energy component as tightly around the high energy 
component as possible without losing any high energy particles. The aperture was chosen to be of radius $35 \mathrm{~mm}$ which was a little larger than the radial co-ordinate of any high energy particle.

As shown by Figure 2, the beam after the aperture plate has a considerable spread of energies intermediate to the two main groups. Although not clearly visible on Fig. 2 , the energy distribution also contains some fine structure caused by the way particles in the RFQ are more likely to be lost at particular places in the r.f. bucket and hence cluster around certain energies.

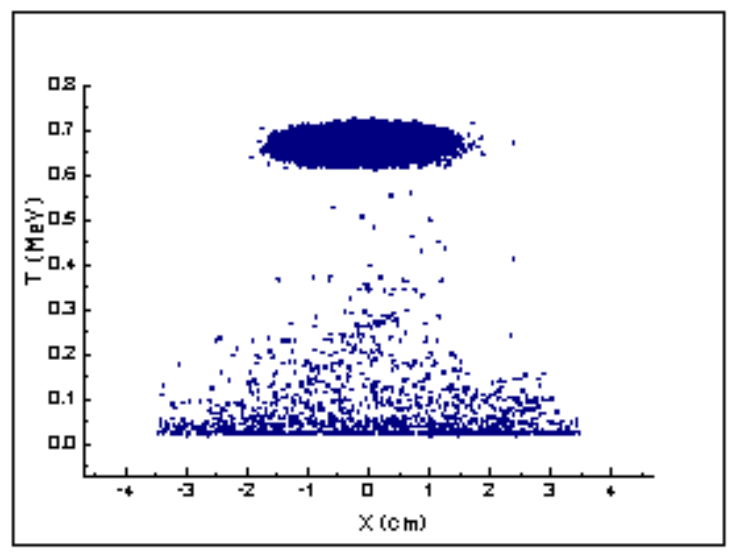

Fig. 2

Plot of $\mathrm{x}$ versus kinetic energy $\mathrm{T}$ for all remaining particles at the start of the parallel plates, with the aperture plate

The voltage to be used on the parallel plates was decided by setting the separation of the plates to be large enough that the high energy beam would not strike them even after being bent, and then trying different voltages in the program written to calculate the effect of the bending field. The final current was to be measured with a beam toroid, whose accuracy was estimated to be no better than $1 \%$, so the system only had to be designed to separate the low energy particles to the order of $1 \%$ of the total number of particles. Using a $45 \mathrm{~mm}$ final aperture throughout, $100 \%$ of high energy particles survived. The results for the low energy particles are in Table 1 below: *

Table 1

\begin{tabular}{ccc}
$\begin{array}{c}\text { Plate Voltages } \\
\text { \% of original low energy } \\
\text { particles remaining }\end{array}$ & $\begin{array}{c}\text { Low energy } \\
\text { particles } \\
\% \text { of total }\end{array}$ \\
\hline
\end{tabular}

$\begin{array}{lll}20 & 6.486 & 0.699 \\ 25 & 4.906 & 0.529 \\ 30 & 3.933 & 0.424\end{array}$

Plate voltages of $\pm 25 \mathrm{kV}$ were chosen because this gives an error in the high energy beam of less than $1 \%$.

Using $\pm 25 \mathrm{kV}$ on the parallel plates with a separation of $70 \mathrm{~mm}$, split left and right 33 and $37 \mathrm{~mm}$ respectively ( non-symmetrical to maintain the highest field while still allowing for the small bending of the high energy component), the bending effect of the plates on the particles was calculated. Plates $140 \mathrm{~mm}$ long were used, because these were as long as possible in the space available while leaving good clearance at either end to avoid sparking between the parallel plates and the aperture and end plates. The clearances were $26 \mathrm{~mm}$ to the aperture plate and $30 \mathrm{~mm}$ to the endplate. In all later calculations, the particles are shown being bent to the right, but this was an arbitrary choice. Figure 3 shows the transverse positions of particles at the toroid if they could pass through the parallel plate without being stopped. Figure 4 shows the calculated distribution of hits on the right hand parallel plate. From this plot the height of the plate from the centre line was chosen to be $60 \mathrm{~mm}$. The nine particles plotted at $\mathrm{x}=-1$ have been reflected by the potential barrier they encountered between the plates.

\subsection{Temperatures of the parallel plate and the aperture plate}

Using this distribution of hits and the distribution of hits on the front face of the aperture plate to estimate the peak number density and hence the peak power density being absorbed by the plates, estimates were made for the maximum temperatures reached by these components in normal operation at $50 \mathrm{~mA}$ ( the maximum expected peak operating current ) for both $1 \%$ and $10 \%$ duty cycles. On the basis of heat loss by radiation only, the maximum temperatures at $10 \%$ Duty Cycle and $50 \mathrm{~mA}$ beam current were $307^{\circ} \mathrm{C}$ for the parallel plate and $233^{\circ} \mathrm{C}$ for the aperture plate. The aperture plate is to be made of $10 \mathrm{~mm}$ Stainless Steel and the parallel plates and their supporting rods have already been made from Copper. The plates are $4 \mathrm{~mm}$ thick.

These estimated temperatures are comfortably below the melting points of these materials.

Figure 5 shows the way the particles of lower energy have been bent to the right and lost on the final aperture. The particles which cannot be separated and which have $\mathrm{x}$ values within the high energy group can also be seen.

\footnotetext{
* The figures in Table 1 are different to those in Table 2 because the calculation in Table 1 did not include the effect on the particles' energies of entering and leaving the region of high potentials between the plates, from and to the region outside, which has zero potential. All other calculations in this paper do include this effect, which was found later in the investigation to be non-negligible.
} 


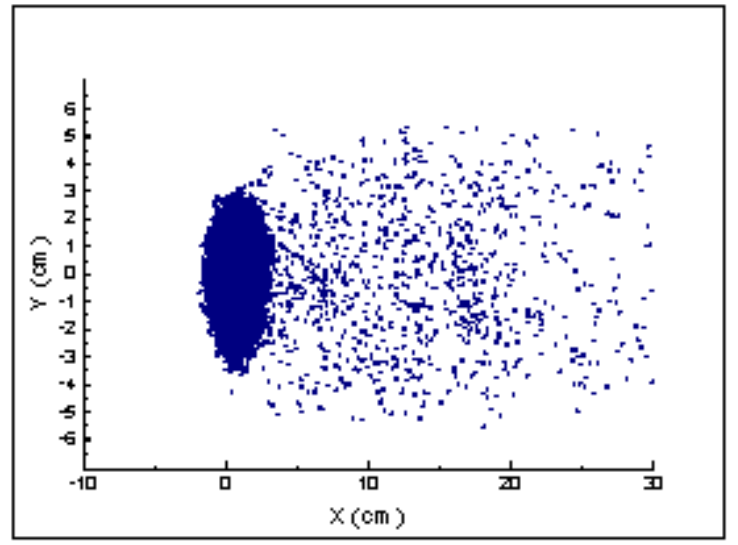

Fig. 3

Transverse distribution of particles just before toroid using $\pm 25 \mathrm{kV} 140 \mathrm{~mm}$ long plates, if no particles were intercepted by the parallel plates

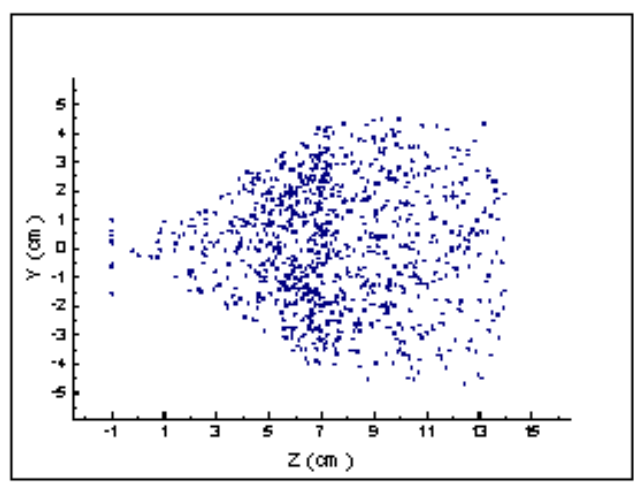

Fig. 4

The distribution of hits on the parallel plate

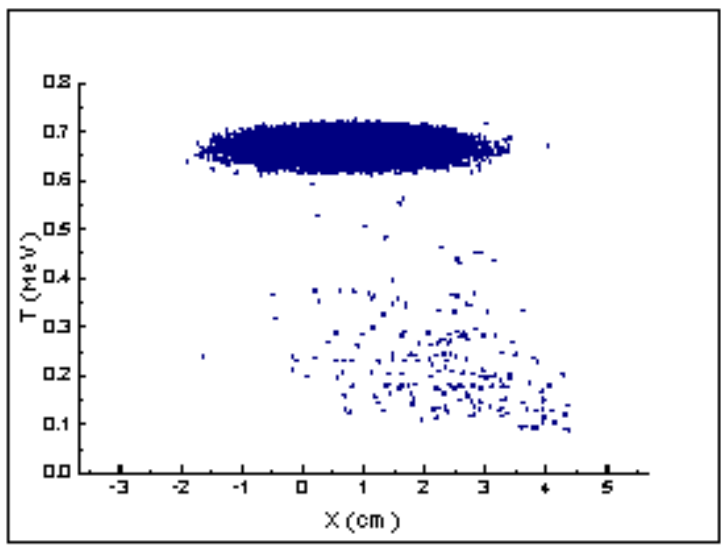

Fig. 5

Plot of $\mathrm{x}$ versus energy $\mathrm{T}$ after being bent and passing through an aperture of radius $45 \mathrm{~mm}$ at the exit of the diagnostics box

\section{RESULTS}

Below in Table 2 are shown the fractions of high and low energy particles remaining at the position of the final toroid which surrounds the exit port of the device for two different exit port radii. If :

$\mathrm{N}_{\mathrm{H}}=$ Number of high energy quasi-particles at end of the device,

$\Delta \mathrm{N}_{\mathrm{H}}=$ Change from original number of high energy particles,

$N_{L}=$ Number of low energy quasi-particles at end of device,

$\Delta \mathrm{N}_{\mathrm{L}}=$ Change from original number of low energy particles, and

$\mathrm{N}_{\mathrm{T}}=\mathrm{N}_{\mathrm{H}}+\mathrm{N}_{\mathrm{L}}=$ original total number of quasi-particles, then:

Table 2

\begin{tabular}{cccccc}
$\begin{array}{c}\text { Aperture } \\
(\mathrm{mm})\end{array}$ & $\mathrm{N}_{\mathrm{H}}\left(\Delta \mathrm{N}_{\mathrm{H}}\right) \%$ high & $\mathrm{N}_{\mathrm{L}}\left(\Delta \mathrm{N}_{\mathrm{L}}\right)$ & $\%$ low & $\frac{\mathrm{N}_{\mathrm{L}}}{\mathrm{N}_{\mathrm{T}}}$ \\
\hline & remaining & remaining $(\%)$
\end{tabular}

\section{CONCLUSIONS}

Simulations indicate that the energy separator system as specified above, i.e. a $35 \mathrm{~mm}$ radius aperture $26 \mathrm{~mm}$ before two parallel plates $140 \mathrm{~mm}$ long, separated by 70 $\mathrm{mm}$, with $\pm 25 \mathrm{kV}$ on them, followed by a drift of $30 \mathrm{~mm}$ and an end aperture of radius $45 \mathrm{~mm}$, should be able to reduce the initial number of low energy particles to $4.8 \%$ of their original number. This represents $0.30 \%$ of the total particle numbers, which is comfortably below the expected accuracy of the toroid which will be used to measure the exit current. This system will produce peak temperatures for $10 \%$ Duty Cycle and $50 \mathrm{~mA}$ beam current, the maximum power that can be envisaged being used, on the parallel plate of about $307^{\circ} \mathrm{C}$, and on the aperture plate of about $233^{\circ} \mathrm{C}$, in the most likely estimate. This system is expected to draw about $0.73 \mathrm{~mA}$ from the power supplies for the parallel plates.

\section{ACKNOWLEDGEMENTS}

My thanks go to Chris Bailey for useful ideas and suggestions, and to Graeme Murdoch for advice on the practical engineering realities and getting the plans drawn up.

\section{REFERENCES}

[1] PARMILA Linac Design Code written by Harunori Takeda, LANL. [2] 'Matching solutions between the ISIS RFQ and Tank 1'- ISIS paper no.ISIS/RFQ/PHYS/JPD/98-2

[3] 'The first order design of the ISIS RFQ Test Stand Spectrometer'ISIS paper no. ISIS/RFQ/PHYS/JPD/98-1 